\title{
EXTRACTION OF TEXTURE FEATURES BY USING GABOR FILTER IN WHEAT CROP DISEASE DETECTION
}

\author{
Mahesh S. Dange ${ }^{1}$, Handore S. M. ${ }^{2}$ \\ ${ }^{1} M E 2^{\text {nd }}$ Year student, E \& TC department, KJ'S Educational Institutes' TCOER, Pune, Maharashtra, India \\ ${ }^{2}$ Associate Professor, E\&TC department, KJ's Educational Institutes TCOER Pune, Maharashtra, India
}

\begin{abstract}
Like country India, there are so many people depending upon agriculture. In this area, many farmers don't know about new diseases which are impacting on their farm. As the disease changes, the disease control policy also changes. So many farmers have very sharp observation on crop diseases, but whenever there is new diseases fall on crops then problems occur. Climate also changes instantly many of times, because of such reasons farmers unable to understand various diseases.

If farmer unable to predict that diseases quickly then it will affect life of crops. Indirectly it gets affects on total productivity of farm. As we are well known about that world facing lot of problems due rapid growth in population. So our goal is to increase agricultural productivity using image processing technology which can help farmer in great extent [7].

In this research work, we are trying that crop disease using Artificial neural network (ANN) which work very effectively. First of all, we have provided an digital image which is taken by digital camera. That image given to Gaussian filter firstly then transferred to adaptive median filter to filter out noise present inside image. Gaussian filter removes Gaussian noise which is present inside image. Adaptive noise filter removes impulsive noise which is present inside image. Also it will reduce distortions which are present inside images. Then image transferred to segmentation part. In image segmentation we have choose CIELAB color space method to extract color components properly. For segmentation we have used Gabor filter. After this we distinguish crop diseases on the basis of texture features which are extracted by Gabor filter [6].
\end{abstract}

Key Words: Artificial Neural Networks, Image preprocessing, Image Acquisition, and Feature Extraction, classification etc...

\section{INTRODUCTION}

India has majority people who are survive on the agriculture. For development we have to pay more attention to increase productivity. Agricultural productivity is depends upon its well planned irrigation systems, crop rotation, use of fertilizers, use of pesticides [2]. Before some years there is stable environment for agriculture, but now days we have realized that climate changes suddenly due to imbalance in their natural cycle. Due to this reasons farmers facing so much problems in prediction of seasons like rainy or winter season. Indian Economy mostly depends on agriculture. Many countries are awakened in research of agriculture like china, US, India, Australia. Seasons are changing so rapidly because of this farmers get so many difficulties in judging environmental changes [7]. Due to this, agricultural products get affected by various diseases. To increase productivity, we have to consider current environmental conditions for farming. Crop diseases will attack whenever this type of unwanted climate changes. For this purpose every farmer must be aware of crop diseases which will be attacked now days.

In this research we have considered one of important cereal i.e. wheat which is taken by farmer mostly. Wheat crop is yielded everywhere in our country. India stands $2^{\text {nd }}$ rank in world for yielding wheat production.

Wheat crop has various diseases like leaf rust, stem rust, loose smut, head blight, stripe rust, septoria, powdery mildew etc [3]. As per analysis, in Asia leaf rust, stripe rust, head blight these diseases take place mostly [1]. In this work we are focused on leaf rust, strip rust, Septoria, Head blight diseases.

1.2 Wheat Crop Common Diseases:

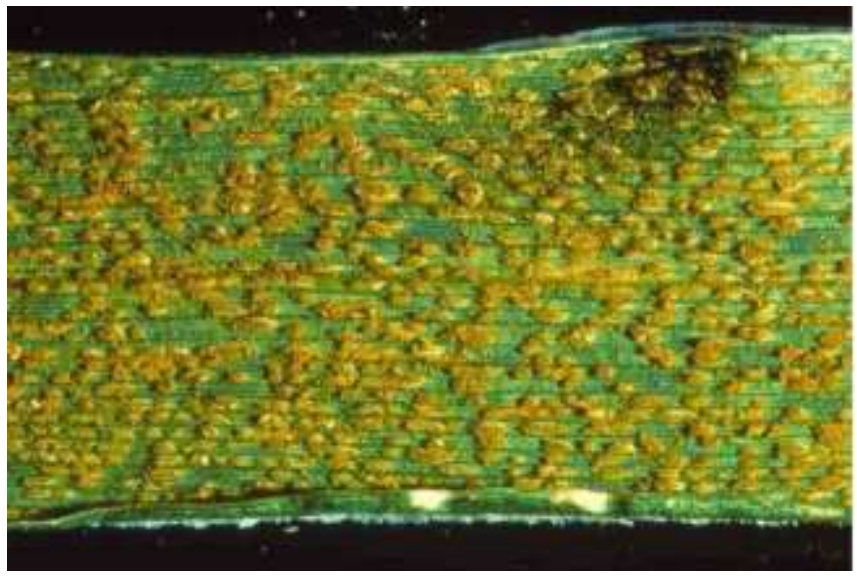

Fig.1. Leaf Rust Image (orange colored heavy dots Or region on leaf). 


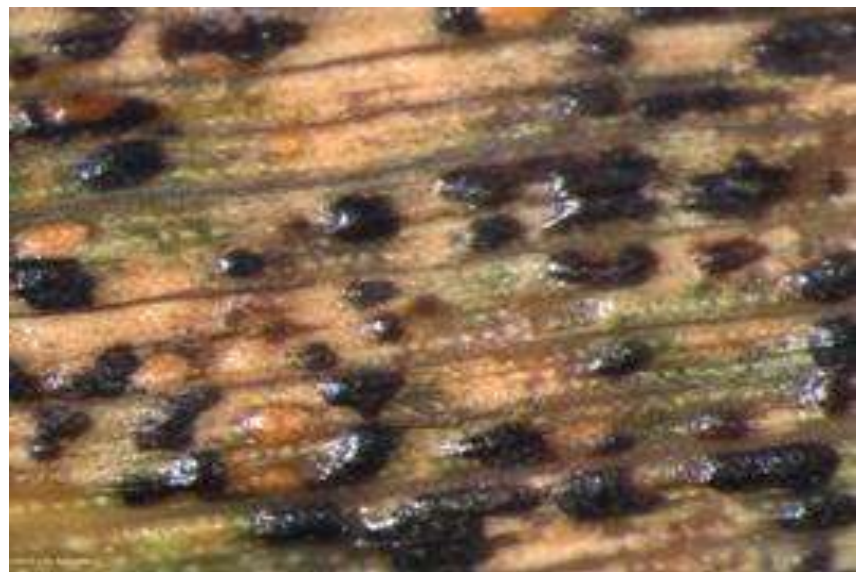

Fig.2. Lose Smut Image (In dark black color)

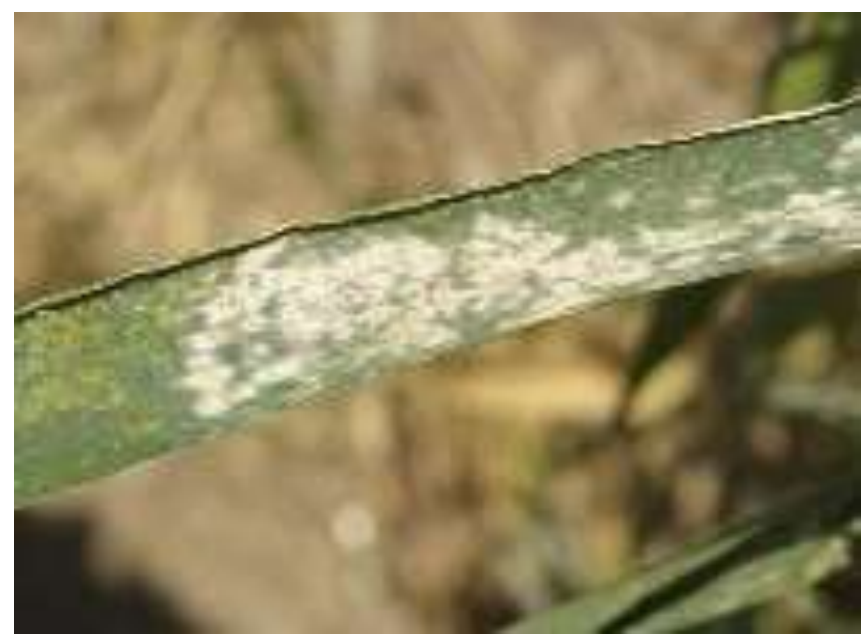

Fig.3. Powdery mildew Image ( white colored dots)

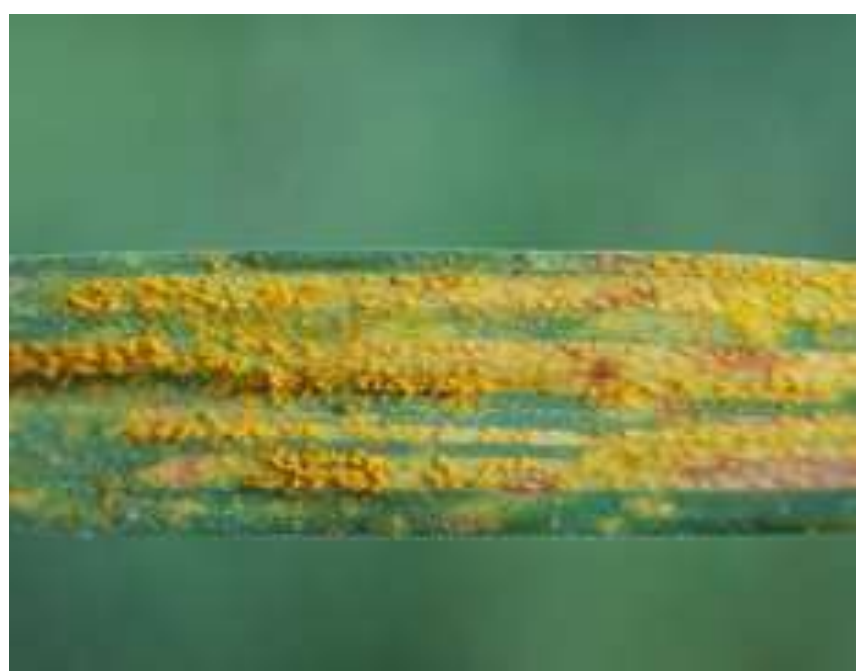

Fig.4. Stripe rust Image

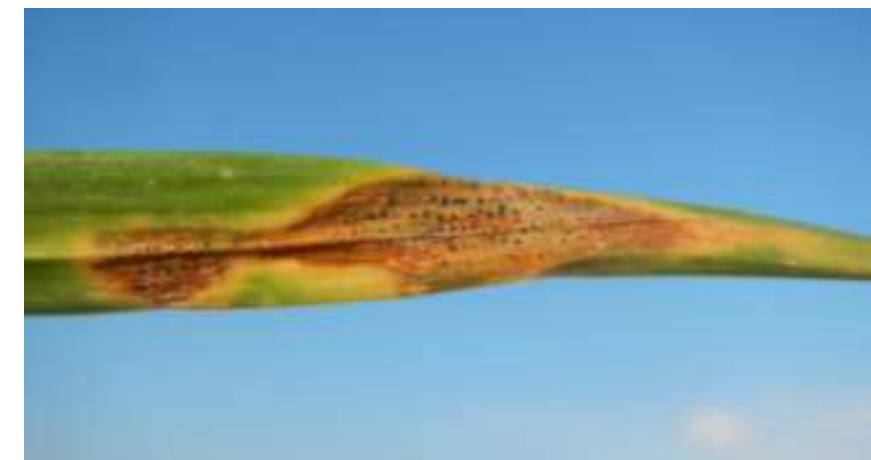

Fig.5. Septoria Image (Light red)

These are the main diseases which will be very common on wheat crop diseases. As per survey result leaf, stem and leaf rust having value $54.7 \%, 19.4 \%$ and $7.7 \%$ diseases on wheat crop field [1].

\section{METHODOLOGY}

This work consists of some basic steps to complete crop disease detection which is represented pictorially in Fig 6.

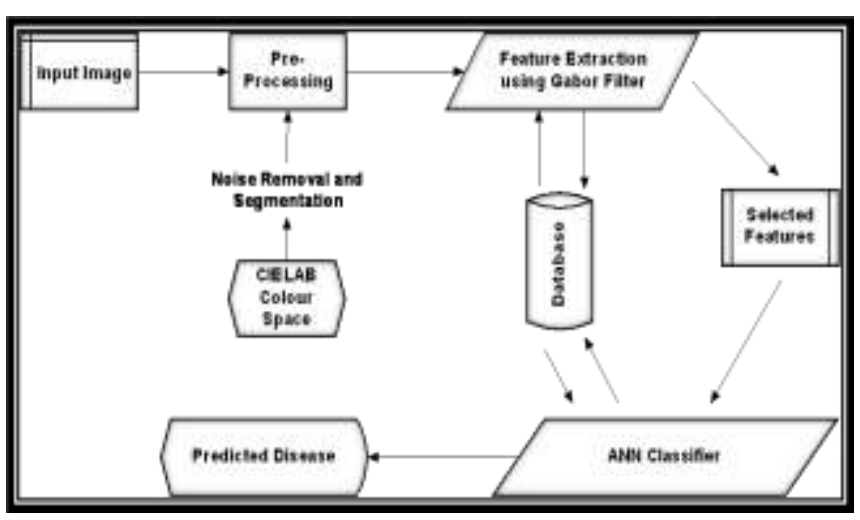

Fig: 6. Block Diagram of Crop Disease Detection

\section{IMAGE PREPROCESSING:}

In this process, we remove Gaussian noise with help of Gaussian filter. Also we are using Adaptive median filter to remove impulsive noise from the input image.

\section{Noiseless Image}

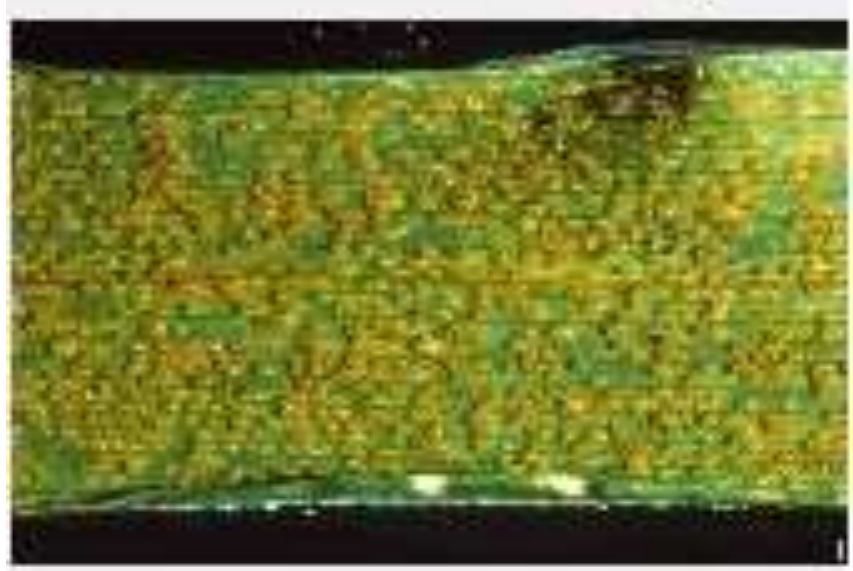

Fig -7. Noiseless Image after Filtering 
From this, we also remove high frequency components values i.e. distortions which are present in input image.

\section{IMAGE SEGMENTATION:}

For this, Image Segmentation using CIELAB color space is performed. Whenever image is converted to CIELAB color space method, at that time we are extracted 3 axes that are, $\mathrm{L}$ axis, $\mathrm{a}^{*}$ axis and $\mathrm{b}^{*}$ axis. $\mathrm{L}$ axis represents intensity or luminance which is present in input image. $\mathrm{L}$ axis has maximum value of 100 which shows perfect reflecting diffuser and $\mathrm{L}$ axis lower limit is zero. $\mathrm{A}^{*}$ axis and $\mathrm{B}^{*}$ axis has no specific limits. Negative value of $\mathrm{a}^{*}$ axis shows green color, and positive value of $\mathrm{a}^{*}$ axis shows red color in image. Also, $b^{*}$ axis positive value indicates yellow color and $y$ axis negative value shows blue color in an image.

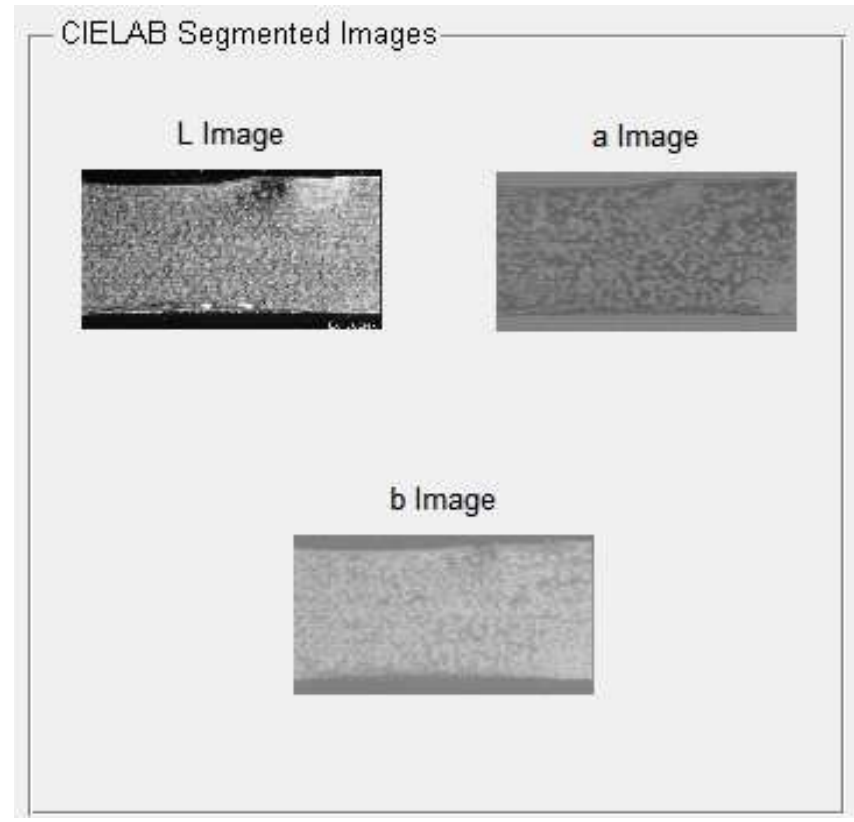

Fig -8: Image Segmentation

\section{FEATURE EXTRACTION:}

In feature extraction, we using Gabor filter to extract texture features of input image. With the help of Gabor filters we are considering GLCM features for our feature extraction of leaf. Area, Perimeter, Contrast, Energy, Homogeneity, major length, minor length, mean of gray level, these are common features we are extracted [5].

\section{Gabor Filter:}

Texture features that are based on the local power spectrum obtained by a bank of Gabor filters are compared [4]. Here we are interesting to extract texture features like Area, Perimeter, Contrast, Energy, Homogeneity, major length, minor length, mean of gray level. The filter is characterized by a preferred orientation and a preferred spatial frequency [4]. When a small-area patch has a wide variation of features of discrete gray tone, the dominant property of that area is texture [6].
Table -1: How Feature Extraction work

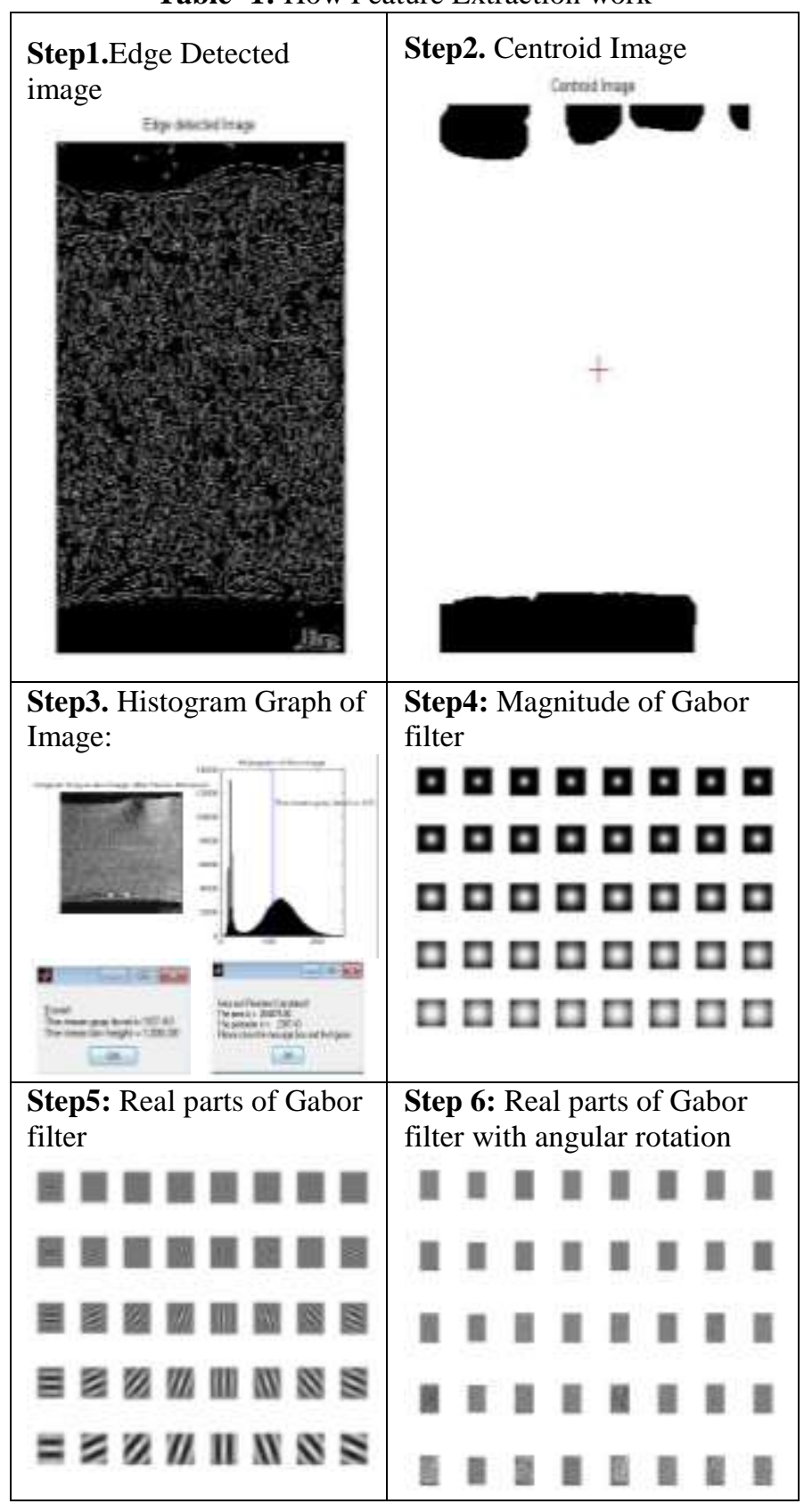

\section{CONCLUSIONS}

In this work, we are come to conclusion that Gabor filter extracts texture features like Area, Perimeter, Contrast, Energy, Homogeneity, major length, minor length, mean of gray level very effectively. Gabor filter also gives GLCM features for feature extraction. In future scope, Extracted features are fed to the classifier (ANN) which is used to predict the crop disease.

\section{REFERENCES}

[1] http://bulletin.ipm.illinois.edu/print.php?id=965

[2] Anand H.Kulkarni, Ashwin Patil R. K., Applying image processing technique to detect plant diseases, International Journal of Modern Engineering Research, vol.2, Issue.5, pp: 3661-3664, 2012. 
[3] John William ORILLO1, Jennifer DELA CRUZ2, Leobelle AGAPITO3, Paul Jensen SATIMBRE4 IraVALENZUELA 5 Identification of Diseases in Rice Plant (Oryza Sativa) using Back Propagation Artificial NeuralNetwork in 7th IEEEInternational Conference Humanoid, Nanotechnology, Information Technology Communication and Control, Environment and Management (HNICEM),The Institute of Electrical and Electronics Engineers Inc. (IEEE) - Philippine Section 12-16 November 2013 Hotel Centro, Puerto Princesa, Palawan, Philippines.

[4] Simona E. Grigorescu, Nicolai Petkov, and Peter Kruizinga Comparison of Texture Features Based on Gabor Filters in IEEE TRANSACTIONS ON IMAGE PROCESSING, VOL. 11, NO. 10, OCTOBER 2002.

[5] NOBUYUKI OTSU, A Threshold Selection Method from Gray-Level Histograms in IEEE TRANSACTIONS ON SYSTREMS, MAN, AND CYBERNETICS, VOL. SMC-9, NO. 1, JANUARY 1979

[6]ROBERT M. HARALICK,K. SHANMUGAM AND IT'SHAK DINSTEIN "Texture Features for Image Classification" in IEEE Transactions on systems, MAN AND CYBERNETICS Vol. SMC-3 No.6 November 1973 pp 610-621.

[7] S. W. Zhang* Y. J. Shang and L. Wang, PLANT DISEASE RECOGNITION BASED ON PLANT LEAF

IMAGE in The Journal of Animal \& Plant Sciences, 25 (3 Suppl. 1) 2015 Special Issue Page: 42-45 ISSN: 1018-7081. 